ORIGINAL ARTICLE

\title{
Fetal echocardiography in trisomy 18
}

D Moyano, I C Huggon, L D Allan

Arch Dis Child Fetal Neonatal Ed 2005;90:F520-F522. doi: 10.1136/adc. 2004.070342

See end of article for authors' affiliations .....................

Correspondence to: D Moyano, Harris Birthright Research Centre for Fetal Medicine, King's College Hospital, Denmark Hill, London, SE5 9RS, UK; Dolores_moyano@yahoo. es

Accepted 20 May 2005 Published Online First 24 May 2005 ....................
Background: Previously reported pathological series suggest that cardiac malformations are universal in trisomy 18. We examined our experience of fetal echocardiography in trisomy 18 for comparison.

Methods: Of 255 fetuses with trisomy 18 detected in our centre between January 1999 and June 2004, 174 were evaluated using fetal echocardiography. Our results were compared to four previous echocardiographic and four autopsy series, comprising 89 and 110 patients, respectively.

Results: Of these 174 fetuses, 114 were examined between 10 and 14 weeks gestation and the remainder between 15 and 33 weeks. An increased nuchal translucency measurement was the reason for referral in most of the early cases and extracardiac anomalies in the remainder. Images were non-diagnostic in 12 cases (7\%), all examined at $<15$ weeks gestation. Abnormal cardiac findings were detected in 118 of the remaining 162 fetuses (73\%), including 15 with functional anomalies. The various heart malformations included ventricular septal defects, tetralogy of Fallot, left heart disease, and atrioventricular septal defects. In all series used for comparison, a similar diversity of disease was seen. In pathological series of trisomy 18, structural heart malformations were found in all cases, but some had lesions which would not be detectable echocardiographically in the fetus.

Conclusion: Abnormal cardiac findings are detectable echocardiographically in the majority of cases of trisomy 18 examined during fetal life, but not in all. A wide spectrum of heart defects is seen. Diagnosis of heart malformations can be made reliably, even in the first trimester at the time of nuchal translucency measurement.
$\mathrm{F}$ etal echocardiography can reliably detect congenital heart disease (CHD) at as early as 12 weeks gestation, at the time of of nuchal translucency (NT) measurement. ${ }^{1}$ The identification of increased NT is associated with a high incidence of chromosomal anomalies. It is well recognised that the types of CHD seen in trisomy 18 are more varied than those associated with trisomy 21 , but conventional wisdom from pathological series suggests that CHD is universal in trisomy $18 .^{2-5}$ As this did not seem to be our experience in trisomy 18 using echocardiography, we examined our data to elucidate this situation.

\section{METHODS}

Our database was searched for cases of trisomy 18 detected in our centre between January 1999 and June 2004. All patients seen in the department sign a consent form for data release, which has been approved by the ethics committee. Of a total of 255 cases, 174 had a detailed cardiac evaluation, 162 of which were diagnostic. All patients were examined transabdominally using an Acuson Aspen system (Acuson, Mountain View, CA) with a 4-7 mHz curvilinear transducer. Of these 174 patients, 114 were examined between 10 and 14 weeks gestation (median 12; crown-rump length between 37.6 and $78.7 \mathrm{~mm}$ ) and the remaining 60 between 15 and 33 weeks, with a mean gestational age for the total group at the time of examination of 15 weeks. A study was considered diagnostic (of normality or abnormality) if the atrioventricular and ventriculoarterial connections could be defined and the relative sizes of the two atria, two ventricles, two great arteries, and the transverse arch and duct could be determined. The reasons for referral for fetal echocardiography are shown in table 1 . The data described in four published echocardiographic series ${ }^{6-9}$ and four pathological series were combined for comparison with our data.

\section{RESULTS}

\section{Current series}

The gestational age was less than 14 weeks in 114/174 (66\%) cases. Images were considered non-diagnostic in 12 cases, all examined at $<15$ weeks. No abnormality of the heart was found in 44 cases, while there were abnormal findings in 118 .

A wide range of CHD was seen and this is illustrated in table 2. According to an accepted cardiological method, each case was assigned only one principal cardiac diagnosis, considered the most fundamental from a cardiological point of view. Some cases had what we grouped together as functional abnormalities. These included isolated tricuspid regurgitation, an abnormal pattern of atrioventricular valve Doppler with a high E/A ratio, or bi-directional flow in either great artery. The E/A ratio is the ratio of the velocity of the passive initial phase of ventricular filling to the active ventricular filling wave during atrial contraction. Normal values have been well described during fetal life. ${ }^{10}$

Of 156 cases with a known NT measurement, the mean measurement was $6.1 \mathrm{~mm}$, with a range of between 0.8 and $12 \mathrm{~mm}$. Of the 156 cases with NT, 127 were over the 99th centile $(81 \%), 10$ were between the 95th and 99th, and 19 were within the normal range (12\%). The mean NT in those with no detectable CHD was the same as the mean NT in those with CHD. Of the 174 patients, 161 proceeded to termination of pregnancy, while there were 10 intrauterine deaths (gestational age range 12-37 weeks) and three neonatal deaths. There are no postmortem data.

\section{Previous echocardiographic series}

In four published series, 89 cases were studied echocardiographically. In two of the series, comprising 57 cases, $^{79}$ examination took place during fetal life at a gestational age range of 14-23 weeks. In the remaining 32 cases, ${ }^{68}$ echocardiography took place after the live birth of an affected infant. Diagnostic categories were grouped and compared to our series as far as was possible and are shown in table 2 .

Abbreviations: CHD, congenital heart disease; NT, nuchal translucency 
Table 1 Reason for referral for fetal echocardiography

\begin{tabular}{lcc}
\hline & \multicolumn{2}{l}{ Gestational age } \\
\cline { 2 - 3 } & $11-14$ weeks & $15-33$ weeks \\
\hline Increased NT & 106 & 2 \\
Extracardiac abns & 7 & 33 \\
Suspected CHD & 0 & 12 \\
Known trisomy 18 & 0 & 7 \\
Miscellaneous & 1 & 6 \\
\hline
\end{tabular}

abns, abnormalities; CHD, congenital heart disease; NT, nuchal translucency;

\section{Previous pathological series}

In four published series, 110 cases were studied at autopsy. ${ }^{2-5}$ Three of the series (a total of 86 cases) described cases which were late stillbirths or were born alive. The remaining series of 24 cases $^{2}$ was a study of fetal material of less than 16 weeks gestation. Diagnostic categories were grouped and compared to our series as far as possible and are shown in table 2 .

\section{DISCUSSION}

Increasingly widespread application of NT screening is presenting the fetal cardiologist with a new high risk group who have a high incidence of chromosomal anomaly and who are being referred for evaluation at much earlier gestations. In this series, the majority of patients were referred at the time of the finding of generally markedly increased NT. A smaller group was evaluated at 20 weeks or later, either because of more modestly increased NT or because of the detection of fetal abnormalities at routine scanning. In $12 \%$ of cases, the NT was within the normal range, a finding which has been previously reported. ${ }^{11}$ Unfortunately from the point of view of auditing our diagnoses, postmortem analyses were available in none of the cases. This is because nearly all the patients were referred from other hospitals and had the termination procedure locally after the chromosome result became available.

In some of the papers used for comparison with this series, there was not sufficient detail for accurate comparison, but an attempt at as fair a comparison as possible was made. CHD was designated in a hierarchical fashion according to the cardiological convention of connection abnormalities taking precedence over additional anomalies, such as ventricular septal defect, and abnormal connections at the atrioventricular junction taking precedence over an abnormal great artery connection. Pathological series in trisomy 18 suggest that CHD is invariable, but close examination of the details in published series show that in some cases only minor CHD, which is not likely to be detectable prenatally, was found. No abnormality was found in the heart in $27 \%$ of our series. It is possible that some examples of minor CHD were overlooked, particularly in the first trimester fetus, but a normal heart was found in $9 / 60$ (15\%) examined in the second trimester, where echocardiography is known to be more accurate. In the two other fetal echo series, no cardiac abnormality was found in $17 \%$ and $55 \%$ of cases, respectively. ${ }^{79}$ Although large or even moderately sized ventricular septal defects can be detected or excluded in early scans, smaller defects would most certainly be overlooked. In the two postnatal echocardiographic series, valvar dysplasia and/ or a ventricular septal defect were reported in all cases, although the septal defect size was sometimes small, and valvar dysplasia mild or confined to one valve. In the only pathology series where the findings are reported in sufficient detail for analysis, ${ }^{5}$ it appears that $9 / 41$ (22\%) cases had abnormalities which would not be detectable echocardiographically prenatally, such as a persistent arterial duct, an atrial septal defect, or a small ventricular septal defect. In addition, valvar dysplasia was commonly described pathologically but only occasionally appreciable echocardiographically, both in this and in the other fetal series. It was more likely to be noticed as an additional finding in the later fetus, rather than in the first trimester fetus, which made up most of our cases. However, some of the functional anomalies, such as tricuspid regurgitation, which were found in the early fetus, may reflect valvar dysplasia. Some of the functionally abnormal findings found in our series seemed to be specific for trisomy 18, such as bi-directional flow in the arterial duct and pulmonary artery or an increased E/A ratio of the atrioventricular valve Doppler, which is abnormal at early gestations.

One reason for any differences between our series and the pathological series in particular, was the timing of examination in gestation. In our series, most patients were studied in the first trimester of pregnancy and, as is known in trisomy 18, many cases would have resulted in intrauterine death. In contrast, in three of the four pathological series, autopsy followed late stillbirth or took place after a live birth. This may be an explanation for the higher incidence of atrioventricular septal defects, for example, in our early fetal series than in the pathological series, and the higher incidence of more minor disease such as small ventricular septal defects in those examined in later pregnancy or after live birth.

In summary, CHD is commonly detected echocardiographically in the fetus with trisomy 18 , but not in every case, as

Table 2 Comparison of the types of CHD found in the present series with the combined data of the four pathological and four echocardiographic series

\begin{tabular}{lccc}
\hline Diagnosis & Present series & $\begin{array}{c}\text { Combined patho- } \\
\text { logical series }\end{array}$ & $\begin{array}{l}\text { Combined echocardio- } \\
\text { graphic series }\end{array}$ \\
\hline Ventricular septal defect & $23(14 \%)$ & $58(52 \%)$ & $39(44 \%)$ \\
Atrioventricular septal defect & $29(17 \%)$ & $2(2 \%)$ & $3(3 \%)$ \\
Double outlet right ventricle & $5(3 \%)$ & $2(2 \%)$ & $4(4 \%)$ \\
Coarctation & $21(13 \%)$ & $21(19 \%)$ & $3(3 \%)$ \\
Mitral atresia & $7(4 \%)$ & $4(4 \%)$ & $1(1 \%)$ \\
Tetralogy of Fallot & $8(5 \%)$ & $11(10 \%)$ & $4(4 \%)$ \\
Hypoplastic left heart & $4(2 \%)$ & $1(1 \%)$ & $3(3 \%)$ \\
Common arterial trunk & $3(2 \%)$ & $4(4 \%)$ & 0 \\
Tricuspid atresia & $1(1 \%)$ & 0 & 0 \\
Pulmonary atresia, IVS & $2(2 \%)$ & 0 & $3(3 \%)$ \\
Aortic stenosis & 0 & $1(1 \%)$ & $2(2 \%)$ \\
Double aortic arch & 0 & $2(2 \%)$ & 0 \\
Functional abnormalities & $15(9 \%)$ & 0 & $5(6 \%)$ \\
No abnormality seen & $44(26 \%)$ & 110 & $22(25 \%)$ \\
Total & 162 & & 89 \\
\hline Only the principal diagnosis was assigned to each case. & &
\end{tabular}




\section{What is already known on this topic}

- Fetal echocardiography can reliably detect congenital heart disease

- Conventional wisdom from pathological series suggests that congenital heart disease is universal in trisomy 18

suggested by pathological series. The forms of CHD which occur in this setting vary widely with ventricular septal defects, atrioventricular septal defects, left heart disease, and tetralogy of Fallot being the most frequent. In practical terms, what has been learnt from this series is that almost any form of complex heart malformation can be associated with trisomy 18. Conversely, a fetus with extracardiac malformations may have trisomy 18 despite the absence of detectable CHD.

\section{Authors' affiliations}

D Moyano, I C Huggon, L D Allan, Harris Birthright Research Centre for Fetal Medicine, King's College Hospital, London, UK

Dr Dolores Moyano is supported by the Fetal Medicine Foundation. The funding source had no role in the study

Competing interests: none declared

\section{REFERENCES}

1 Huggon IC, Ghi T, Cook AC, et al. Fetal cardiac abnormalities identified prior to 14 weeks' gestation. Ultrasound Obstet Gynecol 2002;20:22-9.

\section{What this study adds}

- Abnormal cardiac findings are detectable echocardiographically in most, but not all, cases of trisomy 18 examined during fetal life

- Diagnosis of heart malformations can be made reliably, even during the first trimester at the time of nuchal translucency measurement

2 Hyett J, Moscoso G, Nicolaides KH. Abnormalities of the heart and great arteries in first trimester chromosomally abnormal fetuses. Am J Med Genet 1997:69:207-16

3 Kinoshita M, Nakamura Y, Nakano R, et al. Thirty-one autopsy cases of trisomy 18: clinical features and pathological findings. Pediatr Pathol 1989;9:445-57.

4 Lauweryns JM. Spectrum of clinical and autopsy findings in trisomy 18 syndrome. J Génét Hum 1982;30(1):17-38.

5 Van Praagh S, Truman T, Firpo A, et al. Cardiac malformations in trisomy-18: a study of 41 postmortem cases. J Am Coll Cardiol 1989;13:1586-97.

6 Balderston SM, Shaffer EM, Washington RL, et al. Congenital polyvalvular disease in trisomy 18: echocardiographic diagnosis. Pediatr Cardiol 1990;11:138-42.

7 DeVore GR. Second trimester ultrasonography may identify 77 to $97 \%$ of fetuses with trisomy 18. J Ultrasound Med 2000;19:565-76.

8 Musewe NN, Alexander DJ, Teshima I, et al. Echocardiographic evaluation of the spectrum of cardiac anomalies associated with trisomy 13 and trisomy 18. J Am Coll Cardiol 1990;15:673-7.

9 Shields LE, Carpenter LA, Smith KM, et al. Ultrasonographic diagnosis of trisomy 18: is it practical in the early second trimester? J Ultrasound Med 1998; 17:327-31

10 Tulzer G, Khowsathit P, Gudmundsson S, et al. Diastolic function of the fetal heart during second and third trimester: a prospective longitudinal Dopplerechocardiographic study. Eur J Pediatr 1994;153:151-4.

11 Snijders RJ, Noble P, Sebire N, et al. UK multicentre project on assessment of risk of trisomy 21 by maternal age and fetal nuchal-translucency thickness at 10-14 weeks of gestation. Fetal Medicine Foundation First Trimester Screening Group. Lancet 1998;352:343-6. 